\title{
$94-8$
}

NASA-CR-202591

\section{NAG $3-1375$}

$1 N-0.2-c k$

98067

AIAA 94 - 2576

Measurements of Supersonic Wing Tip Vortices

M.K.Smart, I.M.Kalkhoran and J.Bentson,

Aerospace Engineering Department,

Polytechnic University,

Brooklyn, New York.

\section{8th AIAA Aerospace Ground Testing Conference \\ June 20-23, 1994 / Colorado Springs, CO}




\title{
MEASUREMENTS OF SUPERSONIC WING TIP VORTICES
}

\author{
Michael K. Smart", Iraj M. Kalkhoran+ and James Bentson** \\ Aerospace Engineering Department, \\ Polytechnic University, Brooklyn, New York 11201
}

\begin{abstract}
An experimental survey of supersonic wing tip vortices has been conducted at Mach 2.5 using small scale 4-hole and 5-hole cone probes. The survey was performed 2.25 chords downstream of a semi-span rectangular wing at angles of attack of 5 and 10 degrees. The main objective of the experiments was to determine the Mach number, flow angularity and total pressure distribution in the core region of supersonic wing tip vortices. A secondary aim was to demonstrate the feasibility of using cone probes calibrated with a numerical flow solver to measure flow characteristics at supersonic speeds. Results showed that the numerically generated calibration curves can be used for 4-hole cone probes, but were not sufficiently accurate for conventional 5-hole probes due to nose bluntness effects. Combination of 4-hole cone probe measurements with independent pitot pressure measurements indicated a significant Mach number and total pressure deficit in the core regions of supersonic wing tip vortices, combine $A$ with an asymmetric 'Burger like' swirl distribution.
\end{abstract}

\section{Nomenclature}

M Mach number

$P \quad$ Pressure

Cp Pressure Coefficient

D 5-hole probe nose diameter

q Dynamic Pressure

$\mathrm{x}, \mathrm{y}, \mathrm{z}$ Cartesian coordinates

$\alpha \quad$ Vortex-generator angle of attack

$\theta \quad$ Pitch Angle

$\phi \quad$ Roll Angle

$\xi \quad$ Cone half angle

$v$ Uncertainty

T Swirl Angle $=\tan ^{-1}\left(M_{x} / M_{z}\right)$

$\chi \quad$ Distance from probe tip

\footnotetext{
* Graduate Research Fellow, Student Member AIAA

+ Assistant Professor, Member AIAA

** Industry Professor
}

\section{Subscripts}

$\begin{array}{ll}0 & \text { Settling Chamber } \\ 1 & \text { Total } \\ 2 & \text { Pitot } \\ \infty & \text { Free Stream } \\ \text { av } & \text { Average }\end{array}$

\section{INTRODUCTION}

Many fluid flow problems in aerodynamics and engineering are dominated by vortical structures. These vortical structures, or vortices for short, are the result of the separation and subsequent roll-up of boundary layers which have been forced to leave a surface. Study of the structure and dynamics of such vortex dominated flows is an active area of fluid mechanic research at the present time. Of particular interest to aerodynamicists are the vortices generated by the lifting surfaces of an aircraft. For example, the vortices shed by the wings of in aircraft play an integral part in the generation of lift, and an understanding of their development is important from a general performance standpoint. Also, the vortices shed by the forebody or canards of an aircraft flying at angle of attack can interact with downstream aerodynamic surfaces or be ingested by engine intakes, causing stability and control problems during particular flight manoeuvres. In an effort to expand the experimental data base on supersonic vortex dominated flows, various fundamental vortical interaction studies have been initiated at Polytechnic University 1,2 . These studies have primarily been concerned with the interaction of supersonic wing tip vortices with lifting surfaces and shock fronts. In order to thoroughly analyze the results of these experiments, a parallel program has been undertaken to develop the capability for accurate measurements of the Mach number, total pressure and flow angularity in these vortical flowfields. Multi-hole cone probes have traditionally been the instrument of choice for measurements in threedimensional supersonic flowfields. Pioneering work by Centolanzi ${ }^{3}$, using a $20^{\circ}$ half angle probe with a diameter of $9.5 \mathrm{~mm}(0.38 \mathrm{in})$, showed that 5 -hole cone probes could be used to obtain accurate simultaneous measurements of Mach number, total pressure and flow 
angularity in supersonic flow. The method he reported involved the experimental calibration of a 5-hole probe at numerous pitch and roll angles over a range of Mach numbers. This data was then cleverly cast into calibration curves so that the Mach number, total pressure and flow angularity could be obtained directly from the raw pressure data using an iterative procedure. This technique has become the standard, and numerous investigators 4,5 have reported successful calibration and use of their own cone probes with diameters as small as $1.5 \mathrm{~mm}(0.06 \mathrm{in})$.

The drawbacks of conventional cone probes are their relatively slow time response, of the order of 1 second, and the time consuming experimental calibration procedure. In an attempt to circumvent difficulties associated with slow response time, Naughton et $\mathrm{al}^{5}$ reported the use of a miniature 5-hole probe incorporating fast response piezoelectric pressure transducers inside the wind-tunnel. This probe had a $30^{\circ}$ half angle, a diameter of $1.5 \mathrm{~mm}(0.06 \mathrm{in})$ and was calibrated in the Mach number range 2-4. Their results indicated an improvement in time response of two orders of magnitude over conventional probes, and they used the probe to successfully measure supersonic streamwise vortices with a core size of approximately $4 \mathrm{~mm}(0.16 \mathrm{in})$. The facet of conventional cone probe operation which limits their more widespread use is the experimental calibration procedure. To emphasize this point, a typical calibration of a 5-hole cone probe is summarized below. For a chosen Mach number, the probe is placed at a specified pitch angle, and then rotated about its axis in small increments, collecting the pitot pressure and the four surface pressures at each roll angle. This process is repeated at different pitch angles up to a maximum, and at a few Mach numbers in the range of interest. For typical roll increments of 10 degrees, with 5 different pitch angles and 3 Mach numbers, this amounts to 525 sets of data. This procedure must be performed for each probe of different geometry, and some cursory checking of the calibration should be performed for each similarly shaped probe that is fabricated. It is clear that the amount of wind-tunnel time needed to complete this process is prohibitive for many practical applications.

An alternative to this is the generation of probe calibration curves using a numerical flow solver. In recent years computational fluid dynamics has advanced to the point where the accurate prediction of supersonic flow past smooth pointed bodies is possible at moderate angles of attack. An example of this is reported in references 6 and 7, where a parabolized Navier-Stokes code was shown to accurately predict Mach 8 force and moment data for a $10^{\circ}$ half angle conical body, at angles of attack up to $20^{\circ}$. With particular application to cone probes, it is expected that the flow past a sharp nosed 4-hole cone probe at moderate angles of attack can readily be solved using currently available inviscid conical flow solvers. The flow past a pitched 5-hole cone probe however, which includes a blunted nose for the pitot orifice, would need a full three-dimensional Navier-Stokes solver requiring a significant increase in computational time and effort. Use of numerical solutions to calibrate cone probes is clearly a viable alternative to experiment. Satisfactory calibration of 4-hole cone probes will require the least computational effort, followed by 5-hole probes with surface pressure orifices far from the nose. Calibration of 5-hole cone probes with pressure orifices close to the nose will be the most difficult to accomplish.

The main objective of the current investigation was to determine the Mach number, total pressure and swirl distributions in the core region of supersonic wing tip vortices. In order to accomplish this task, both a 4hole cone probe and a 5-hole cone probe were commercially acquired. A half angle of $\xi=30$ degrees was chosen for both probes and each had a diameter of $3.2 \mathrm{~mm}$ ( $0.125 \mathrm{in})$, which was the smallest available size. The calibration curves used for the cone probes were generated using computational solutions instead of the conventional experimental calibration. Cone probe surveys of the tip vortices generated by a rectangular half-wing at $\alpha=5$ and 10 degrees angle of attack are presented in this report. The results of these surveys add to the scarce amount of experimental information on supersonic wing tip vortices currently available in the literature $8,9,10$. Such data is important to the increased understanding of supersonic vortical flows and for use as input to numerical computations. A discussion of the use of numerically generated calibration curves is also included in this work due to the original nature of this approach.

\section{EXPERIMENTAL PROGRAM}

\section{Wind Tunnel and Test Conditions}

The current investigation was conducted in Polytechnic University's $15 \times 15$ in $^{2}$ supersonic blowdown wind tunnel facility. ${ }^{11}$ It is an intermittent blowdown wind tunnel with a square test section of 38.1 $\mathrm{cm} \times 38.1 \mathrm{~cm}(15$ in $\times 15$ in) and is capable of producing unit Reynolds numbers in the range of $26 \mathrm{x}$ $10^{6}$ to $22 \times 10^{7}$ per meter $\left(8 \times 10^{6}\right.$ to $66 \times 10^{6}$ per foot) over a Mach number range from 1.75 to 4.0. The experimental studies reported here were conducted at a nominal test section Mach number of 2.5 . The stagnation pressure and temperature for these experiments were $0.45 \mathrm{MPa}(65 \mathrm{psia})$ and $290 \mathrm{~K}$ 
respectively, resulting in a unit Reynolds number of 4.3 $x 10^{7}$ per meter $\left(1.3 \times 10^{7}\right.$ per foot). A typical test time for the experiments was three seconds.

\section{Experimental Arrangement}

A schematic of the experimental arrangement is shown in figure 1 . The vortex generator was a rectangular half-wing with a diamond shaped cross section ( 8 degrees half angle), a chord length of 50.8 $\mathrm{mm}(2 \mathrm{in})$, and a span of $165 \mathrm{~mm}$ (6.5 in). The vortex generator was mounted vertically at the base of the test section with angle of attack capability between 0 and 10 degrees. The cone probes were mounted in a $25.4 \mathrm{~mm}$ (1.0 in) diameter tube which protruded vertically through the test section ceiling. The probes were situated with their tips approximately $108 \mathrm{~mm}$ (4.5 in) or 2.25 vortex generator chords downstream of the halfwing trailing edge, and were able to be traversed in the vertical direction. A wedge shaped fin with 18 degree included angle was installed at the leading edge of the support tube for structural support of the cone probes and to isolate the probes from any upstream influence of the support tube bow shock.

\section{Cone Probes}

Two small scale cone probes were used in for the current study, dimensioned sketches of which are shown in figure 2. Each probe had a diameter of 3.2 $\mathrm{mm}(0.125 \mathrm{in})$ and a half angle $\xi=30$ degrees. The 5 -hole cone probe included four equally spaced static pressure orifices on the cone surface (denoted a, b, c and d), together with a total pressure orifice placed centrally on a blunted nose (denoted e). The 4-hole cone probe included four equally spaced static pressure orifices on the cone surface but had negligible nose bluntness. The probes had a $90^{\circ}$ elbow $50.8 \mathrm{~mm}(2.0$ in) behind the tip in order to reduce the line length between the pressure orifices and the transducers to 36 $\mathrm{cm}$ (14 in). The pressure lines in the probe tips and the pressure orifices on the probes were $0.38 \mathrm{~mm}$ $(0.015 \mathrm{in})$ in diameter. A line length of $36 \mathrm{~cm}$ (14 in) at this diameter was expected to degrade probe response time to an unacceptable level for typical test times of 3 seconds. To circumvent this problem, the internal diameter of the pressure lines was increased to $0.64 \mathrm{~mm}(0.025 \mathrm{in})$ approximately $25 \mathrm{~mm}(1 \mathrm{in})$ behind the elbow. Benchtop tests indicated that the time response of the cone probes to instantaneous pressure changes of the order of 1 atmosphere was approximately 0.5 seconds. The spherical co-ordinate system shown in figure 3 is used for the current work. The $z$ direction is parallel with the free stream and the probe axis, while the $x$ and $y$ directions are horizontal and vertical respectively. The sign convention for the pitch angle $\theta$ and the roll angle $\phi$ are as shown in figure 3. The cone probes were manufactured with a permanent roll angle of $\phi=5^{\circ}$, so the surface pressure orifices a,b,c and d were at circumferential angles 5 , 275,185 and 95 degrees respectively.

\section{Instrumentation and Data Acquisition System}

Five Kulite pressure transducers (model ITQ$1000-50 \mathrm{~A}$ ) were used in the experiments. These had a range from $0-345 \mathrm{kPa}(0-50 \mathrm{psia})$ and a natural frequency response of $12 \mathrm{KHz}$. Output from the transducers was first amplified by Honeywell Accudata 122 DC amplifiers and then digitised using a Metrabyte das-16, 12-bit analog-to-digital converter board at a rate of $500 \mathrm{hz}$ per channel for a period of three seconds. The experimental error associated with typical cone surface and pitot pressures reported in this paper is $v_{p}$ $= \pm 0.25$ psia, and a conventional uncertainty analysis indicated that the uncertainty in test section Mach number and cone surface pressure coefficient are $v_{M}$ $= \pm 0.018$ and $v_{C p}= \pm 0.03$ respectively. Shadowgraphs were taken of the flow using a spark light source which provided micro-second range exposure times. Multiple spark shadowgraphs of the flowfield were possible at a rate of two per second.

\section{COMPUTATIONAL CALIBRATION OF THE CONE PROBES}

As already described, the calibration of cone probes has traditionally required an exhaustive amount of experimental data taken with the probe at different pitch and roll angles, over a range of Mach numbers. In the present study the cone probe calibration curves were generated using a computational solution. These solutions were obtained using a Navier-Stokes solver obtained from F.Marconi of Grumman Aerospace. This code uses a computational algorithm based on Beam and Warming's approximate factorization ${ }^{12}$ in conjunction with Roc's flux difference splitting ${ }^{13}$. The solution of the equations is accomplished using an upwind alternate direction implicit technique similar to that of Thomas ${ }^{14}$. In the present study it was found that for the Reynolds numbers considered, the solutions obtained by running the code as an Euler solver agreed well with the Navier-Stokes solutions. Thus all the data presented here was obtained from inviscid computations.

The process of numerically generating a full set of calibration curves for a cone probe with specified half angle is as follows. Firstly, a conical grid must be 
generated to match the probe geometry. For the current work the $81 \times 63$ grid used was sheared to the leeward side to capture all shocks. Computational runs can then be completed for each combination of Mach number and pitch angle in the range of interest. Note that each run calculates the complete flow past the probe, so that the full circumferential pressure distribution can be extracted from a single run. The 525 sets of cone surfaces pressures required for the typical experimental calibration described above can be obtained with 15 runs. For the current work the Mach number range of interest was between Mach 1.75 and 2.5 , and the maximum pitch angle was $20^{\circ}$. These values represent the anticipated Mach numbers and maximum flow angularity in the core region of supersonic wing tip vortices. Calibration curves were generated for $\mathrm{M}=1.75,2.0,2.25$ and 2.5 at pitch angle increments of 5 degrees. A typical calibration curve for Mach 2.5 is shown in figure 4. After Centolanzi ${ }^{3}$, the surface pressure data at each $\theta$ and $\phi$ is plotted versus $C_{p \eta}=\left(P_{d}-P_{b}\right) / q_{\infty}$ and $C_{p \xi}=\left(P_{a}-P_{c}\right) / q_{\infty}$. It is generally found ${ }^{3,5}$ that when experimental 5 -hole probe data is plotted in this way, little variation with Mach number occurs. This was also the case for the numerically generated data used in the current work. The pointed cone assumption made in the numerical model means that no calculation of the pitot pressure is performed in the numerical solution at different pitch angles and Mach numbers. Results of both Centolanzi ${ }^{3}$ and Naughton et $\mathrm{al}^{5}$ showed that between Mach 1.5 and 4 , the pitot pressure measured by a 5-hole cone probe corresponds well with the theoretical stagnation pressure behind a normal shock for pitch angles up to $20^{\circ}$. Hence the Raleigh pitot formula is used in the current work to complete the data needed for a full cone probe calibration. Figure 5 shows a graph of $\mathrm{P}_{\mathrm{a} v} / \mathrm{P}_{2}$ ( where $P_{a v}=\left(P_{a}+P_{b}+P_{c}+P_{d}\right) / 4$ ) versus Mach number calculated using the numerical method and the Raleigh pitot formula.

The iterative procedure for determining the Mach number, total pressure and flow angularity from the pitot and surface pressures measured by a 5 -hole probe is fully described in references 3 and 5 . In short, the flow Mach number is estimated from the ratio $\mathrm{P}_{\mathrm{av}} / \mathrm{P}_{2}$, and then combined with the measured cone surface pressures to calculate $C_{p \eta}$ and $C_{p \xi}$. The two Euler angles, $\phi$ and $\theta$, can then be determined from calibration maps such as figure 4 . In general, iteration is required because $P_{a v} / P_{2}$ varies with $\theta$, hence after a first estimate of Mach number and flow angularity has been made, the Mach number must be adjusted for this variation. It is usually found ${ }^{3,5}$ that only one or two iterations are required. A plot of the ratio $P_{a v} / P_{2}$ versus $\theta$ calculated by the numerical scheme at Mach
2.5 is shown in figure 6. Negligible variation of $\mathrm{P}_{\mathrm{av}} / \mathrm{P}_{2}$ is observed, which was the case for all the Mach numbers included in the calibration, hence no iteration of the Mach number is required. Determination of the flow properties in the current work was therefore reduced to a one step procedure. In summary, the determination of flow properties using the numerically generated calibration curves is identical to the conventional procedure, except that no iteration is required.

\section{EXPERIMENTAL RESULTS AND DISCUSSION}

\section{Initial Test Results}

The initial phase of the experimental program was concerned with establishing the accuracy and time response characteristics of the cone probes. Figure 7 shows the normalized pressure versus time traces for the 4-hole probe with nominal test section Mach number of 2.5 and $\theta=20^{\circ}$. The pressure recorded at all orifices are observed to reach a steady state value after approximately 0.75 seconds. This result was typical of both the 4-hole and 5-hole probes, indicating that the time response of the probes are acceptable for use in short duration blowdown tunnels. In general, the initial one second of a typical three second run was discarded during data processing. Satisfactory use of the 4-hole and 5-hole cone probes depends entirely on the accuracy with which the numerical solution predicts their surface pressure distribution. A comparison between the numerical and experimental results at Mach 2.5 is shown in figure 8 , with the probes at $\theta=$ $0,5,10,15$ and 20 degrees. It can be seen that except for a slight under prediction of the peak pressure on the windward side of the probe, the numerical scheme predicts the 4-hole cone probe surface pressure with high accuracy. The surface pressures measured on the 5-hole probe however, differed from both the 4-hole probe and numerical results, particularly on the leeward side where a significantly greater suction was observed. The generally lower surface pressures observed for the 5-hole cone probe at small pitch angles are consistent with those reported in the literature where a blunted nose has been found to cause an over-expansion in the region local to the nose, followed by an asymptotic approach to the pointed cone results. Krasnov ${ }^{15}$ supplied a universal curve for predicting the scale of the over-expanded region on blunted cones at zero angle of attack. For a flat nosed cone with $\xi=30^{\circ}$ at Mach 2.5, this curve predicts that the pressure coefficient on the cone surface approaches $99 \%$ of the pointed cone result at $\chi / D=3.22$ (where $\chi$ is the downstream distance measured from the flat nose). 
The pressure orifices on the 5-hole probe used in this study are at $\chi / D=0.83$, which is well within the region expected to be affected by nose bluntness. The effect of nose bluntness on cones at angle of attack is not as easy to quantify, but it is not surprising that the 5-hole probe shows significant over-expansion due to its blunt nose, particularly on the leeward side. Based on these results, it is expected that calibration curves generated by the conical Euler solver can be used with confidence for the sharp nosed 4-hole cone probe. However, numerically generated calibration for the blunted 5 -hole probe would require a fully three-dimensional numerical scheme and a significant increase in computational time and effort.

Limitation of the current work to the 4-hole cone probe introduces an additional complexity to the determination of the Mach number, total pressure and now angularity. That is, the pitot pressure must be measured independent of the cone surface pressures. In order to fully determine the properties at a point, two measurements are required; a 4-hole probe measurement (supplying the cone surfaces pressures) and a separate pitot pressure measurement. This doubles the number of runs needed to complete a survey and also introduces the geometrical complexity of making sure both measurements are taken at the same position in the flow. For the surveys presented here, the 5-hole probe was used simply as a pitot probe. The use of the conical Euler solver to generate cone probe calibration curves has required a doubling of the number of runs in return for significant economy gains in the probe calibration. This difficulty may be overcome by using a three-dimensional computational scheme which properly treats the nose bluntness.

A shadowgraph of the flow taken during a typical cone probe survey of the tip vortex generated by the half-wing at $\alpha=10^{\circ}$ is shown in figure 9(a). Flow is from left to right and the vortex core can be clearly seen convecting downstream from the tip of the halfwing towards the cone probe. Figure 9(b) shows a shadowgraph of the same flow but with the leading edge fin removed from the probe support tube. Here the intersection of the vortex with the detached bow shock on the support tube is belicved to generate the observed large scale vortex distortion which renders the cone probe useless. Similar vortex distortions were reported in reference 1 for the head-on interaction of vortices with wedge leading edges. This figure vividly illustrates one of the difficulties which can be encountered during intrusive measurements of vortical flows. The vortex distortion was removed by addition of a leading edge fin (18 included angle) which generates a significantly weaker shock than the support tube, while still supplying the required structural support. An image of the $\alpha=10^{\circ}$ tip vortex generated by a laser light sheet technique is shown in figure 10 . The sheet was projected normal to the flow direction approximately 3 chords downstream of the half-wing. The vortex core can be clearly seen in the image surrounded by an asymmetric swirling structure which joins up with the half-wing wake in the lower portion of the image.

\section{Vortex Surveys}

Cone probe surveys were conducted for the wing tip vortices generated by the half-wing at $\alpha=5$ and 10 degrees. In this work, the vortex generated by the half-wing at $\alpha=5$ degrees will be called the weak vortex, and that generated by the half-wing at $\alpha=10$ degrees will be called the strong vortex. The survey of each tip vortex was conducted 2.25 half-wing chords downstream of its trailing edge in a spanwise (vertical) line through the axis of each vortex. The lateral position of the vortex axes is defined as the position at which the pitot pressure was observed to be a minimum. The upwash of each vortex is then the lateral distance from the trailing edge of the half-wing to the position of minimum pitot pressure. The upwash for the weak and strong vortices was measured to be $3.1 \mathrm{~mm}(0.12 \mathrm{in})$ and $3.3 \mathrm{~mm}(0.13 \mathrm{in})$ respectively. Spanwise pitot pressure surveys through the axis of each vortex are shown in figure 11. The spanwise position of the vortex axes is defined as the position of minimum pitot pressure once again, and the inwash of each vortex is then the spanwise distance between the half-wing tip and the vortex axis. Noting that the wing tip is $165 \mathrm{~mm}$ (6.5 in) above the base of the test section, the inwash of the weak and strong vortices are $1.3 \mathrm{~mm}$ (0.05 in) and $3.5 \mathrm{~mm}$ (0.14 in) respectively. As can be seen from figure 11, significant pitot pressure deficit relative to the freestream is observed for both tip vortex strengths. For the weak vortex, the pitot pressure ratio dips to a minimum of $\mathrm{P}_{2} / \mathrm{P}_{0}=0.27$ and approaches the freestream value of $\mathrm{P}_{2} / \mathrm{P}_{0}=0.503$ approximatcly $9.7 \mathrm{~mm}(0.38 \mathrm{in})$ above the half-wing tip. Some effect of the wake may be seen at the lower limit of the survey where the pitot pressure ratio continues to decrease below its freestream value. For the strong vortex the pitot pressure ratio is seen to reach a minimum of $\mathrm{P}_{2} / \mathrm{P}_{0}=0.175$ and approaches the freestream value $19 \mathrm{~mm}(0.75 \mathrm{in})$ above the half-wing tip. Some evidence of a wake is also observed in a similar fashion to the weak vortex. In general, the magnitude and spatial scale of the pitot pressure deficit increases with half-wing angle of attack.

Figures 12(a), (b) and (c) show the spanwise Mach number distributions for both vortex strengths. 
A single step procedure based on that by Centolanzi ${ }^{3}$ was used to calculate these values from the raw pressure data. Since significant variation in the pitot pressure occurs over lengths of the order of the cone probe diameter $(3.2 \mathrm{~mm})$, the pitot value used to calculate the flow properties was averaged over a $2 \mathrm{~mm}$ $(0.08 \mathrm{in})$ length centered on the cone probe tip. Figure 12(a) shows the distribution of lateral Mach number $\left(M_{x}\right)$ for both vortices. For the geometry of the current experiments, $M_{x}$ may be interpreted as the swirl component of the Mach number. The distributions show a similarity to the classic Burgers swirl velocity profile with an inner linear swirl distribution, surrounded by a region with swirl similar to an irrotational vortex. The point of zero swirl was found to correspond closely with the point of minimum pitot pressure (figure 11) for both vortices. It is noted however that the profiles are not symmetric, but show a larger swirl and core radius outboard of the half-wing (see also figure 10). This asymmetry is typical of wing tip vortices. The average core diameter for the weak and strong vortices was observed to be $6.7 \mathrm{~mm}(0.26 \mathrm{in})$ and $8.0 \mathrm{~mm}(0.32 \mathrm{in})$ respectively. Figure 12 (b) shows the distribution of spanwise Mach number $\left(M_{y}\right)$ for both vortices. These distributions indicate that the vortex cores were not travelling parallel with the freestream at the survey position, but were being washed inwards. Further evidence of this may be obtained by close examination of figure $9(a)$, where the tip vortex core can be clearly seen to be washed inwards from the half-wing tip. The magnitude of the inwash indicated in Figure 12(b) increased with halfwing angle of attack, and peaked close to the vortex axes for both cases. Figure 12(c) shows the distribution of streamwise Mach number $\left(M_{z}\right)$ for both vortices. Significant Mach number deficit is observed to occur for both vortices in a small region near their respective axes. Outside this region, which is of the same spatial scale as the vortex core, $M_{2}$ is close to $M_{\infty}$. The wakelike $\mathrm{M}_{\mathrm{z}}$ profiles reach a minimum of $\mathrm{M}_{\mathrm{z}}=1.70$ and 1.53 for the weak and strong vortices respectively. Streamwise Mach number deficits of this magnitude have not been previously reported for supersonic wing tip vortices and have significant implications for vortex interaction studies.

Based on the results presented in Figures 12(a) and (c), the magnitude of the swirl angle $\tau$ in the vortices is plotted in figure 13. As noted earlier, the vortices are not axi-symmetric and the peak swirl angle occurred outboard of the half-wing tip. The maximum swirl angles are $\tau=7.4^{\circ}$ and $8.7^{\circ}$ for the weak and strong vortices respectively. The spanwise total pressure $\left(\mathrm{P}_{1}\right)$ distributions for the weak and strong vortices are shown in figure 14 . As expected, significant total pressure deficits occur in the core regions, the minimum total pressure ratios being $\mathrm{P}_{1} / \mathrm{P}_{\mathrm{o}}=0.33$ and 0.19 respectively for the weak and strong vortices. Also of note is the absence of any effect of the shockexpansion wave structure generated by the half-wing, which indicates that the survey position 2.25 chords downstream of the half-wing trailing edge is within the 'test diamond' in the current experiments. In summary, the supersonic wing tip vortices exhibited many characteristics commonly found in low speed wing tip vortices, including an asymmetric 'Burger like' swirl distribution and significant total pressure deficits. To the authors' knowledge the substantial streamwise Mach number deficit observed in the vortex core regions have not been previously reported for supersonic wing tip vortices. The spatial scale and the strength of the vortices was observed to increase with half-wing angle of attack.

\section{CONCLUSIONS}

Cone probe surveys were conducted at Mach 2.5 for the wing tip vortices generated by a rectangular half-wing at 5 and 10 degrees angle of attack. The tip vortices exhibited many characteristics similar to their low speed counterparts, including asymmetric 'Burger like' swirl distributions and significant total pressure deficits. A wake-like streamwise Mach number distribution was observed for both cases, and the scale and strength of the vortices increased with half-wing angle of attack. These results add to the small amount of experimental supersonic tip vortex data available in the literature. Computational calibration of commercially available cone probes using an Euler solver was found to be satisfactory for pointed 4-hole probes. Extension of numerical calibration to 5-hole cone probes would require a treatment of the nose bluntness in the numerical model, which was not attempted here. The current use of computational fluid dynamics to calibrate cone probes is a viable alternative to conventional experimental calibration, particularly when wind-tunnel time is at a premium.

\section{Acknowledgements}

This work was supported by the Air Force Office of Scientific Research under Grant F49620-93-10009 and by NASA Lewis Research Center under Grant NAG3-1378. Computing resources for the work presented here was provided by the Pittsburgh Supercomputing Center. The authors are grateful to Frank Marconi for many useful discussions and for providing the computer code used to generate the calibration curves. The assistance of Mr Lester Orlick, 
Mr Kuo-Kuang Liu and Mr Joe Zammit was greatly appreciated during the work.

\section{REFERENCES}

1. Kalkhoran,I.M., "Vortex Distortion During Vortex-Surface Interaction in a Mach 3 Stream ${ }^{n}$, AIAA Journal, Vol 32, No. 1, January 1994.

2. Kalkhoran, I.M., and Sforza, P.M., "Airfoil Pressure Measurements During Oblique Shock WaveVortex Interaction in a Mach 3 Stream," AIAA Journal, Vol. 32, No. 4, April 1994.

3. Centolanzi,F.J, "Characteristics of a $40^{\circ}$ Cone for Measuring Mach Number, Total Pressure and Flow Angles a Supersonic Speeds", NACA-TN-3967, May 1957.

4. Andrews,D.R, and Sawyer,W.G, "The Calibration of a $60^{\circ}$ Cone to Measure Mach Number, Total Pressure, and Flow Angles at Supersonic Speeds", Aeronautical Research Council CP-628, London, 1962. 5. Naughton,J.W, Cattafesta, L.N, and Settles, G.S, "Miniature, Fast-Response 5-Hole Conical Probe for Supersonic Flowfield Measurement", AIAA Journal, Vol. 31, No. 3, March 1993.

6. Oberkampf,W.L, and Aeschliman,D.P, "Joint Computational/Experimental Aerodynamics Research on a Hypersonic Vehicle, Part 1: Experimental Results, AIAA Journal, Vol. 30, No. 8, August 1992.

7. McWherter Walker,M., and Oberkampf,W.L, "Joint Computational/Experimental Aerodynamics Research on a Hypersonic Vehicle, Part 2: Computational Results, AIAA Journal, Vol. 30, No. 8, August 1992.

8. Davis,T., "The Measurement of Downwash and Sidewash Behind a Rectangular Wing at Mach 1.6", Journal of Aeronautical Science, May 1952, p329.

9. Adamson,D. and Boatright, W.B., "Investigation of Downwash, Sidewash and Mach number Distribution Behind a Rectangular Wing at Mach 2.41", NACA TR 1340, 1950.

10. Wang,F.Y., and Sforza,P.M., "An Experimental Investigation of Tip Vortices at Mach 2.5", AlAA paper 93-3448, 1993.

11. Kalkhoran, I.M., Cresci, R.J., and Sforza, P.M., "Development of Polytechnic University's Supersonic Wind Tunnel Facility," AIAA Paper 93-0798, January 1993.

12. Beam,R.M. and Warming,R.F., "An Implicit Factored Scheme for the Compressible Navier-Stokes Equations", AIAA Journal, Vol. 16, No. 4, 1978.

13. Roe,P.L., "Discrete Models for the Numerical Analysis of Time-Dependent Multi-dimensional Gas Dynamics", J. Comp. Phys., Vol. 63, pp 458-476, 1986. 14. Thomas,J.L. and Walters, R.W., "Upwind
Relaxation Algorithms for the Navier-Stokes Equations", AIAA Journal, Vol. 25, No. 4, pp 527-534, 1987.

15. Krasnov, N.F, "Aerodynamics of Bodies of Revolution", American Elsevier Publishing Company, New York, 1970. 


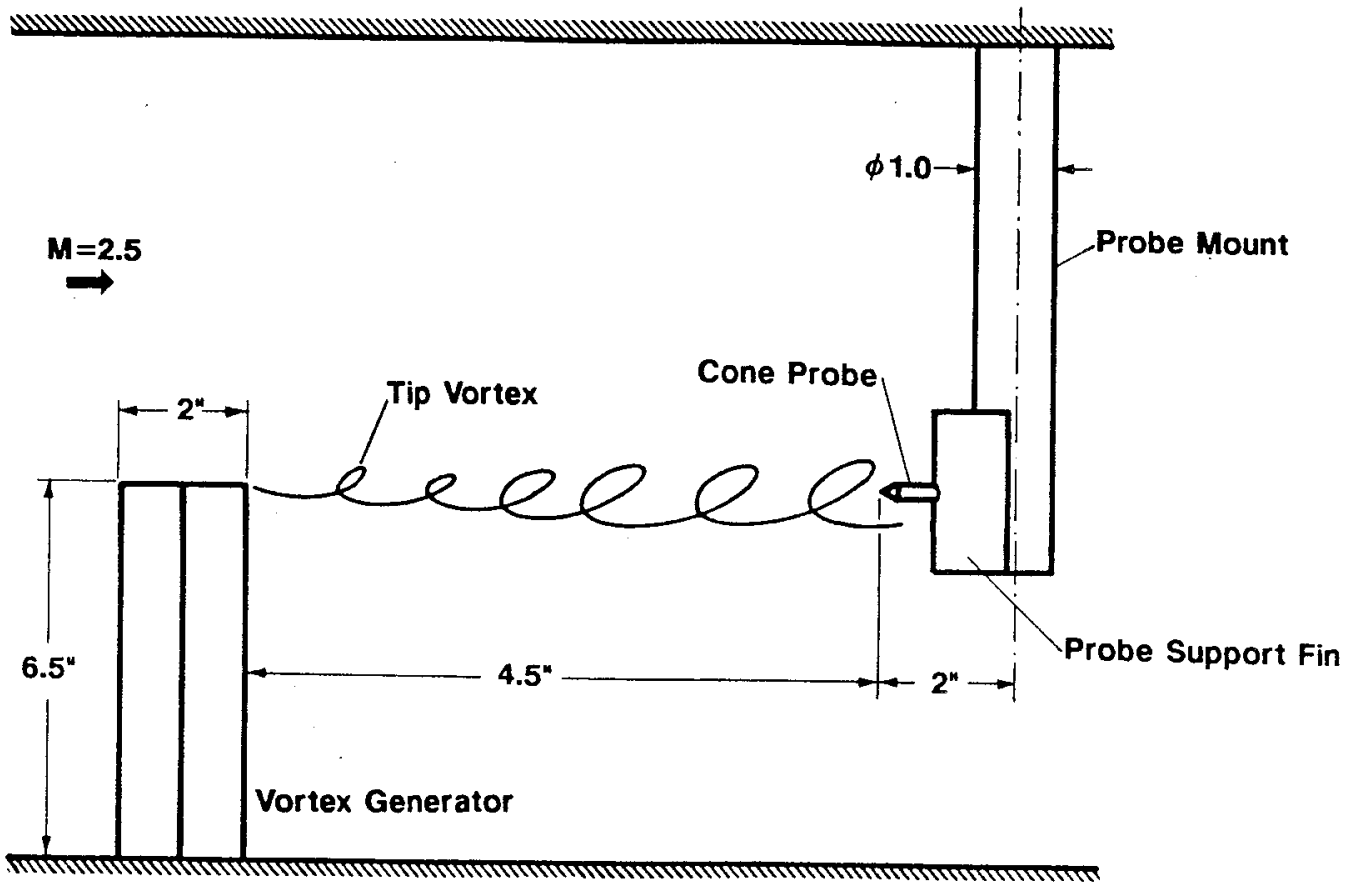

Figure 1 - Schematic of Experimental Arrangement.
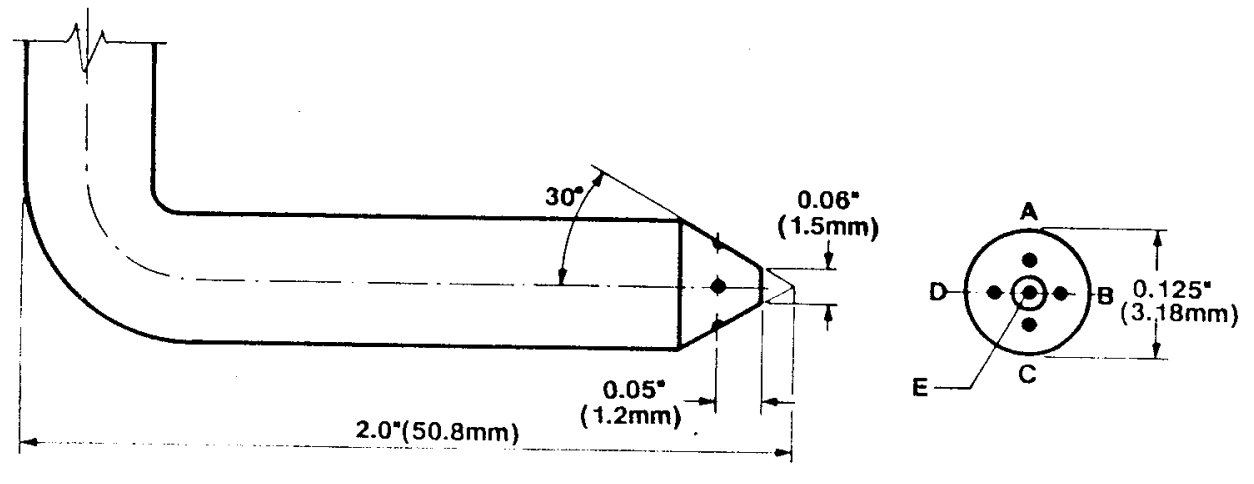

(a) 5 Hole Probe

Head-on View
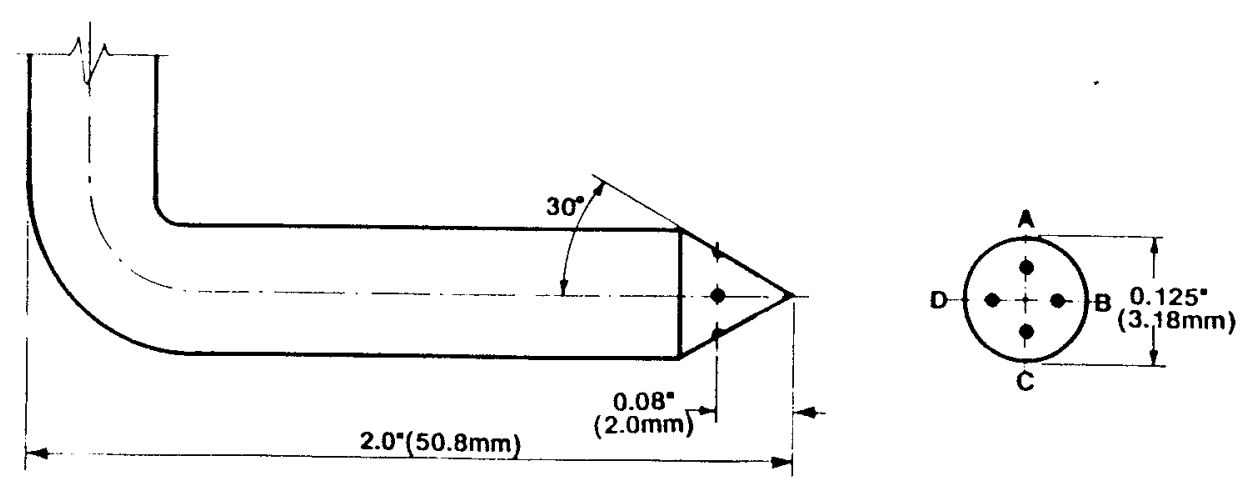

(b) 4 Hole Probe

Side View

Head-on View

Figure 2 - Cone Probes 


\section{Front view}
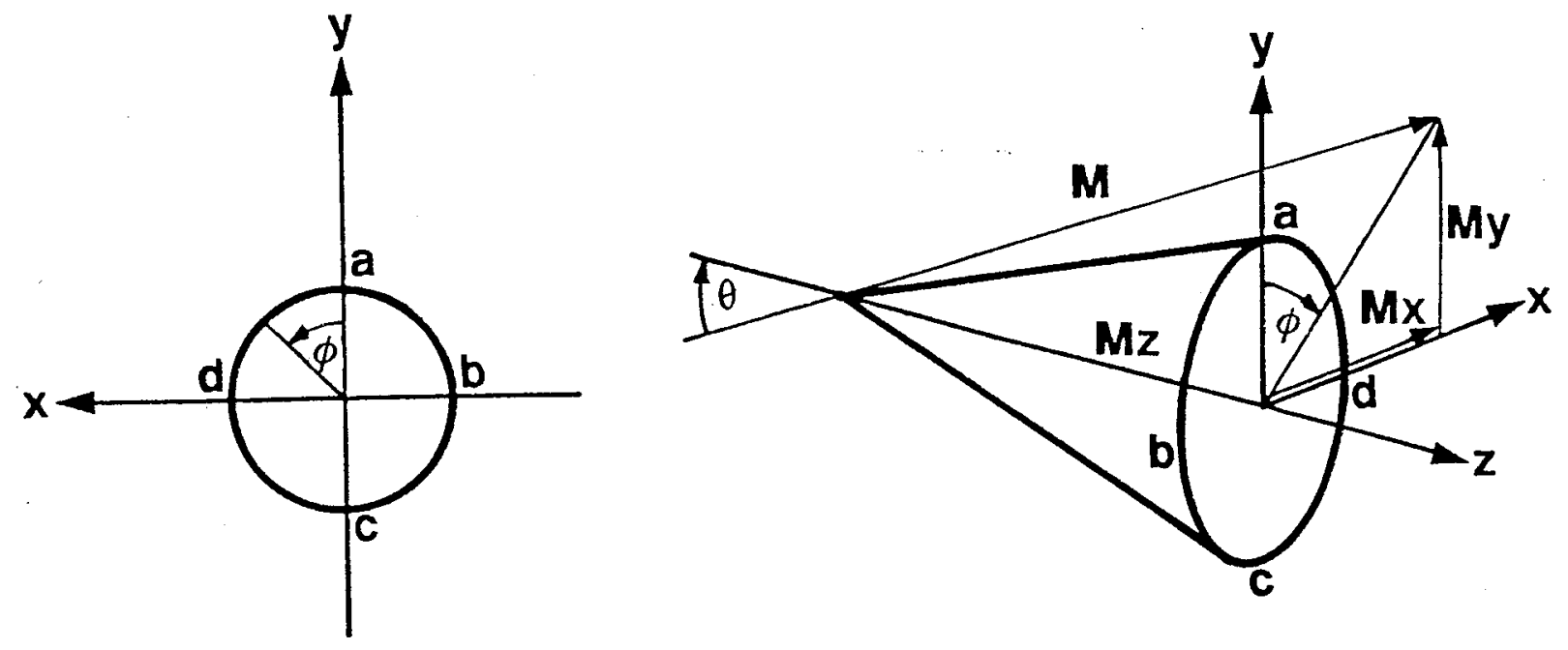

Figure 3 - Spherical Co-ordinate System.

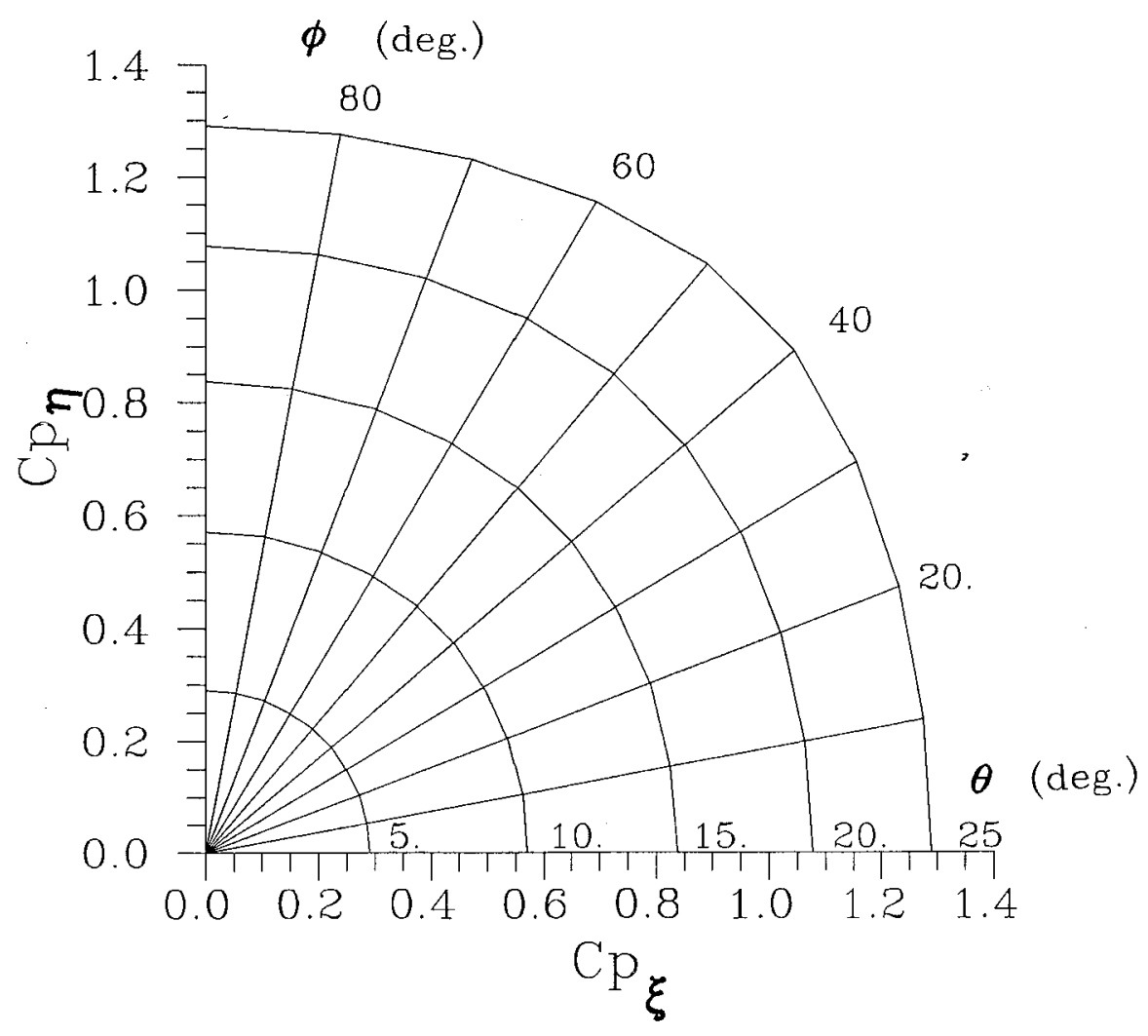

Figure 4 - Calibration Map for $\mathrm{M}=2.5$. 

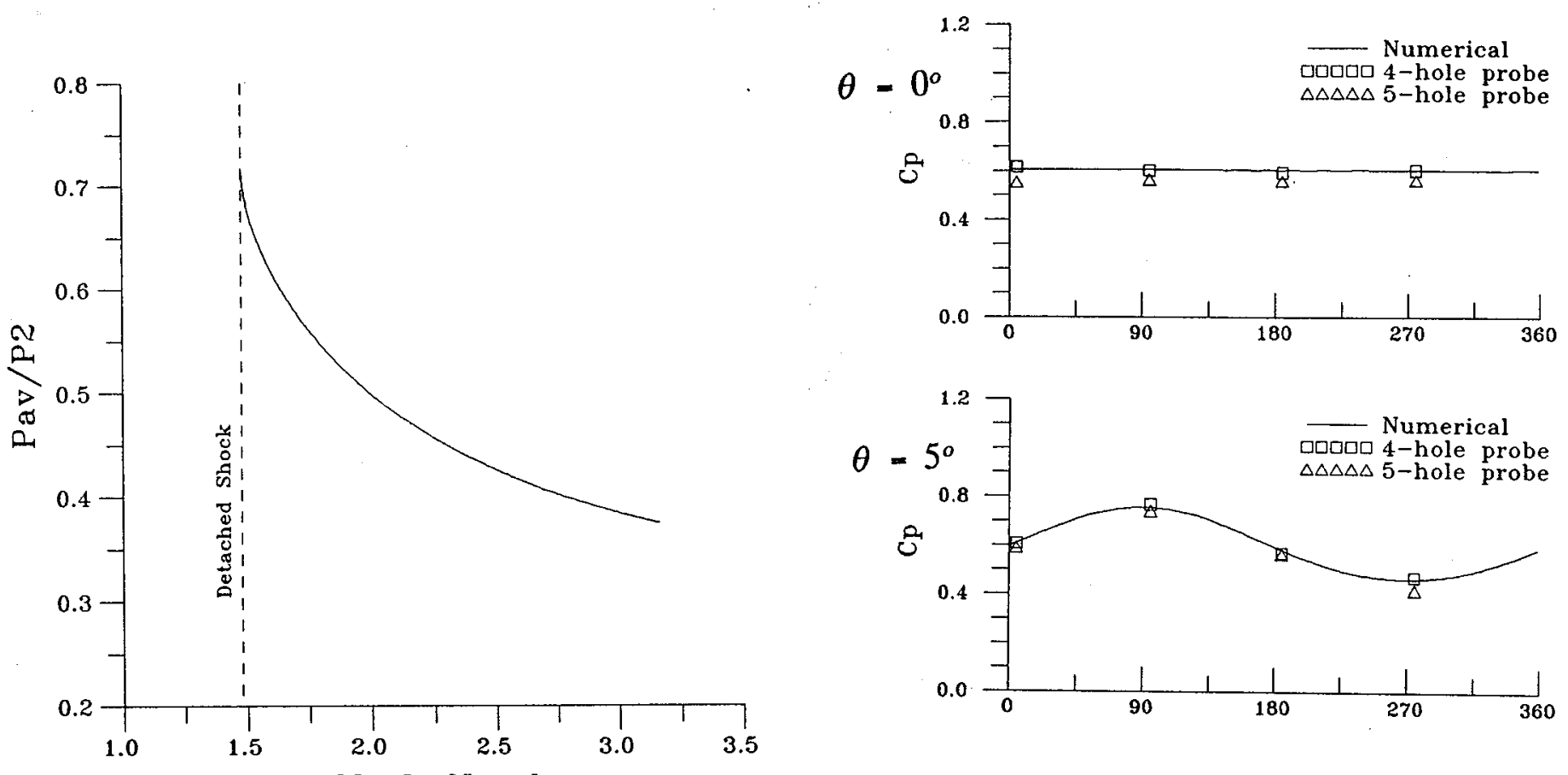

Mach Number

Figure $5-\mathrm{P}_{\mathrm{av}} / \mathrm{P}_{2}$ versus Mach Number.
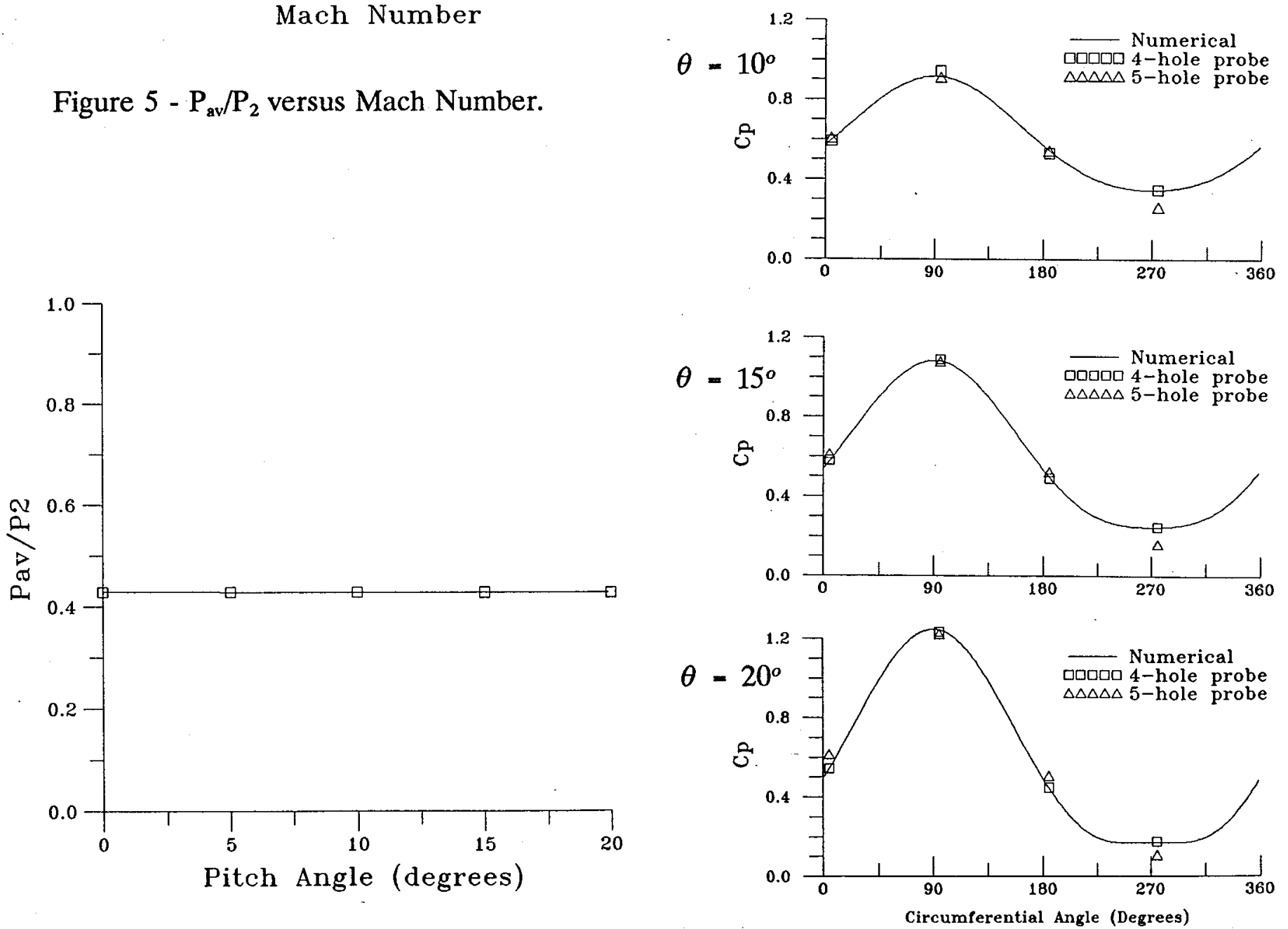

Figure $6-P_{a v} / P_{2}$ versus pitch angle at $M=2.5$.

Figure 8 - Circumferential Cp Distributions at $\mathrm{M}=2.5$. 

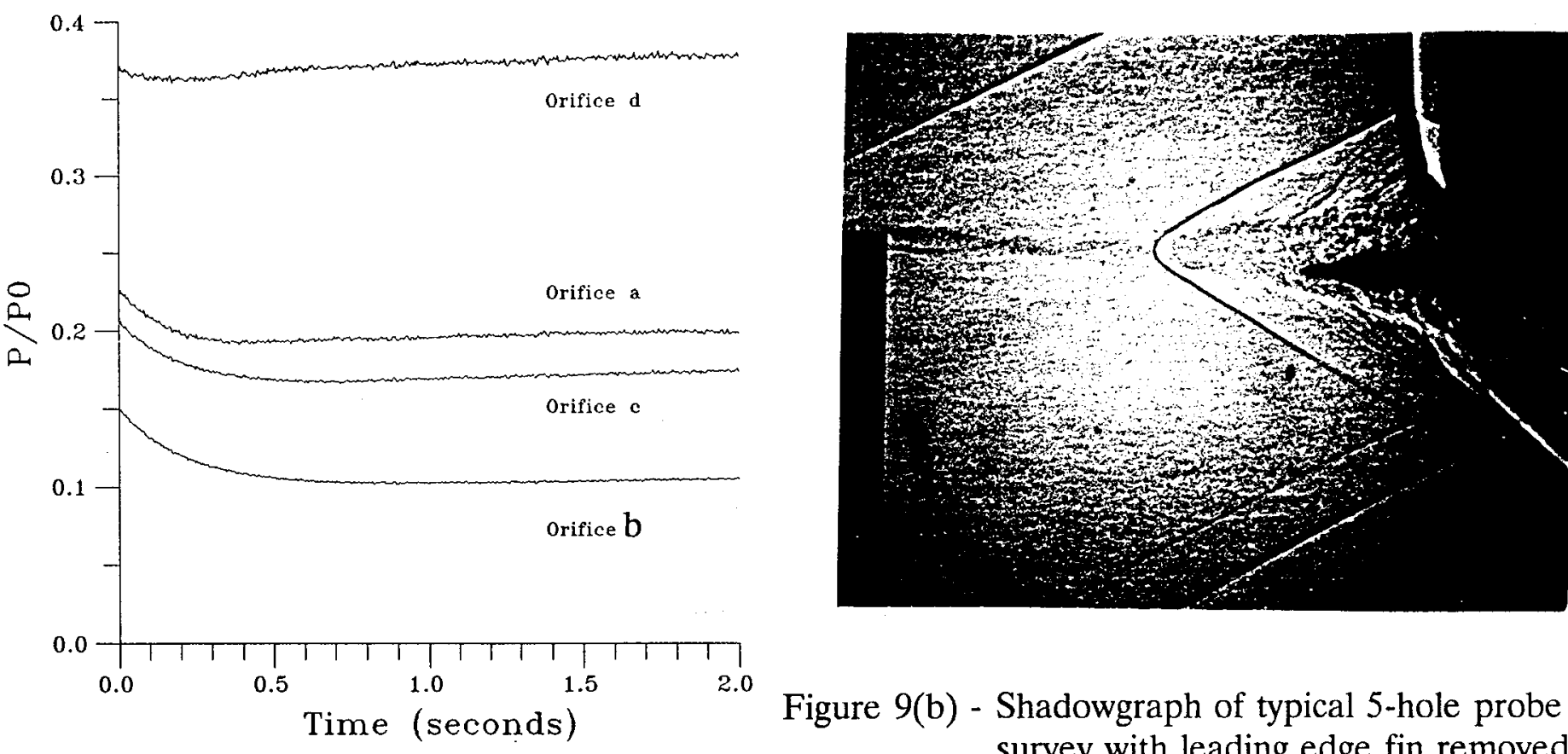

Figure 9(b) - Shadowgraph of typical 5-hole probe survey with leading edge fin removed.

Figure 7 - Pressure versus time for a typical run.

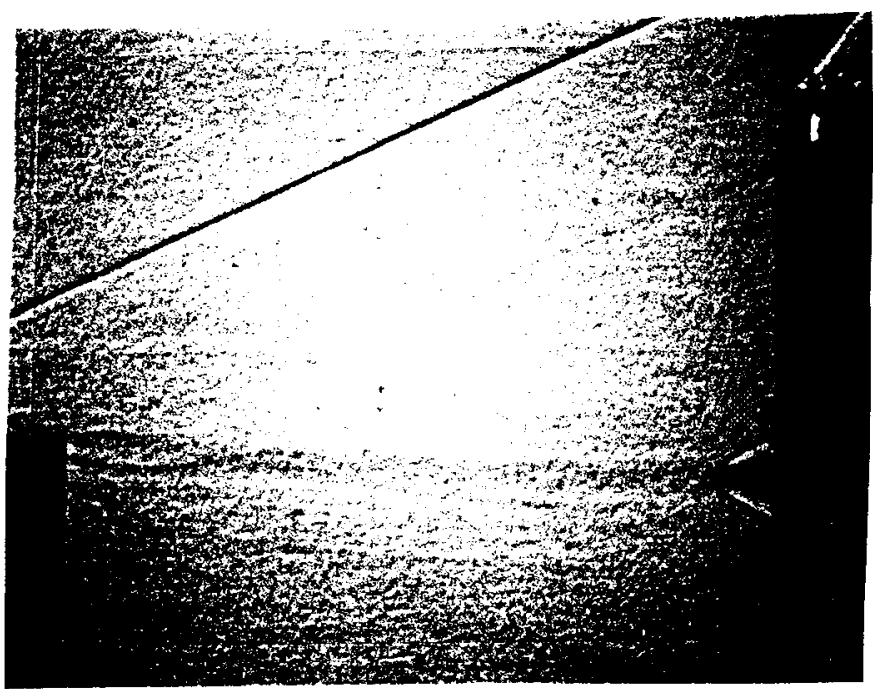

Figure 9(a) - Shadowgraph of typical 5-hole probe survey.

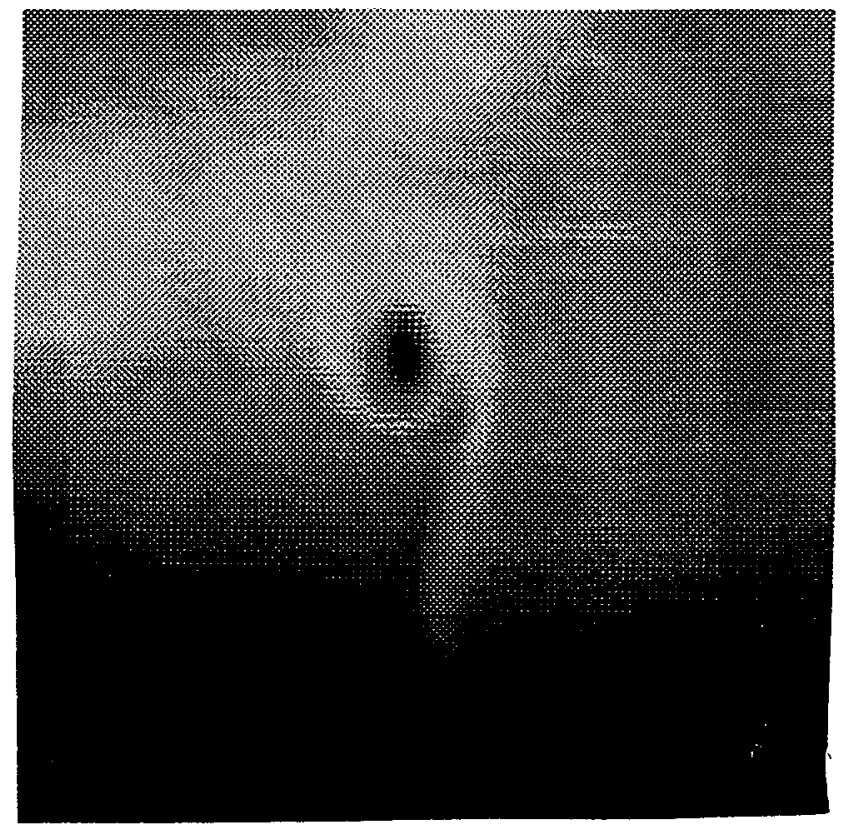

Figure 10 - Laser light sheet image of $\alpha=10^{\circ}$ vortex. 


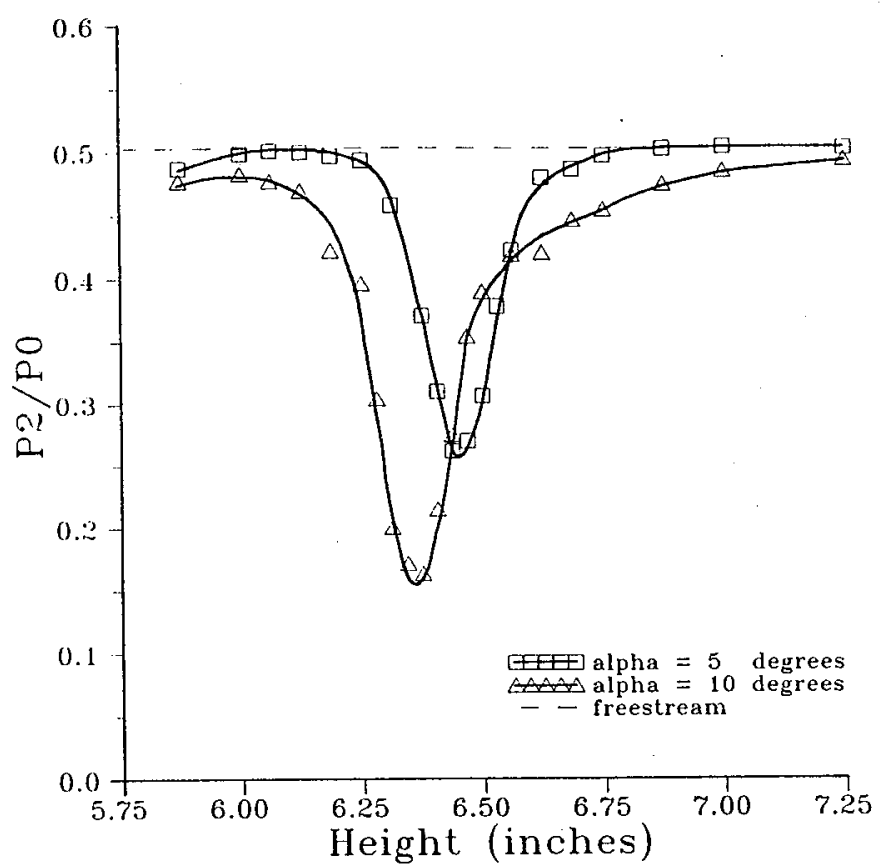

Figure 11 - Pitot Pressure Distribution

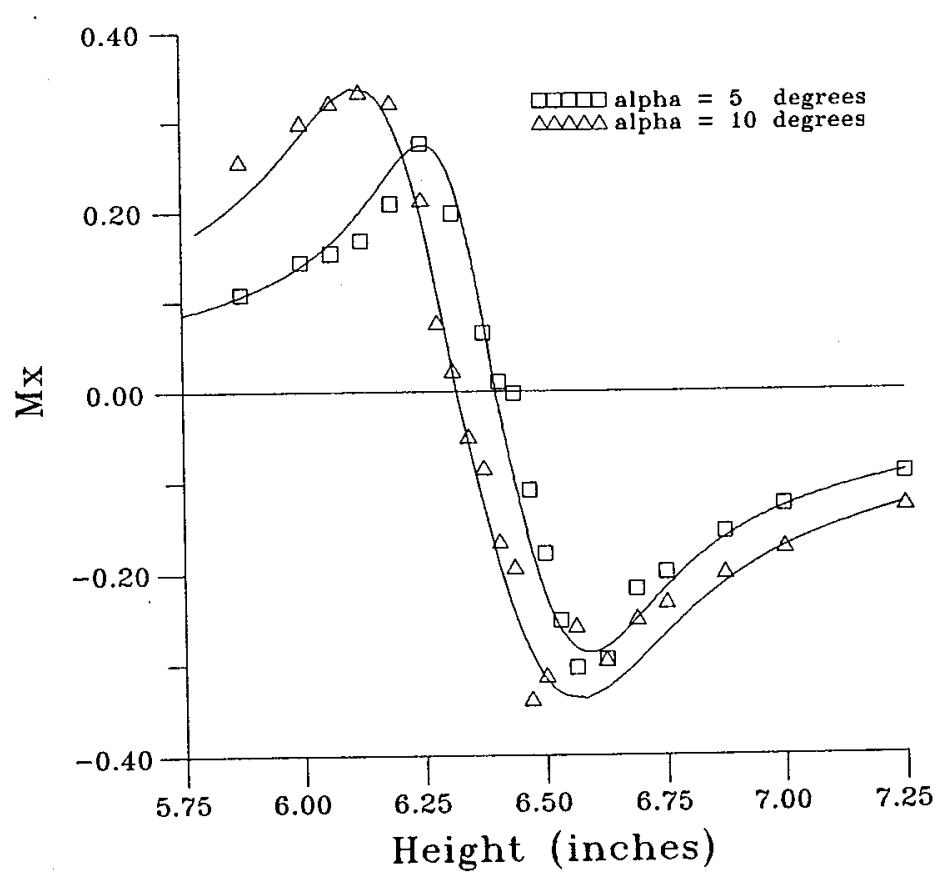

Figure 12(a) $-M_{x}$ Distribution

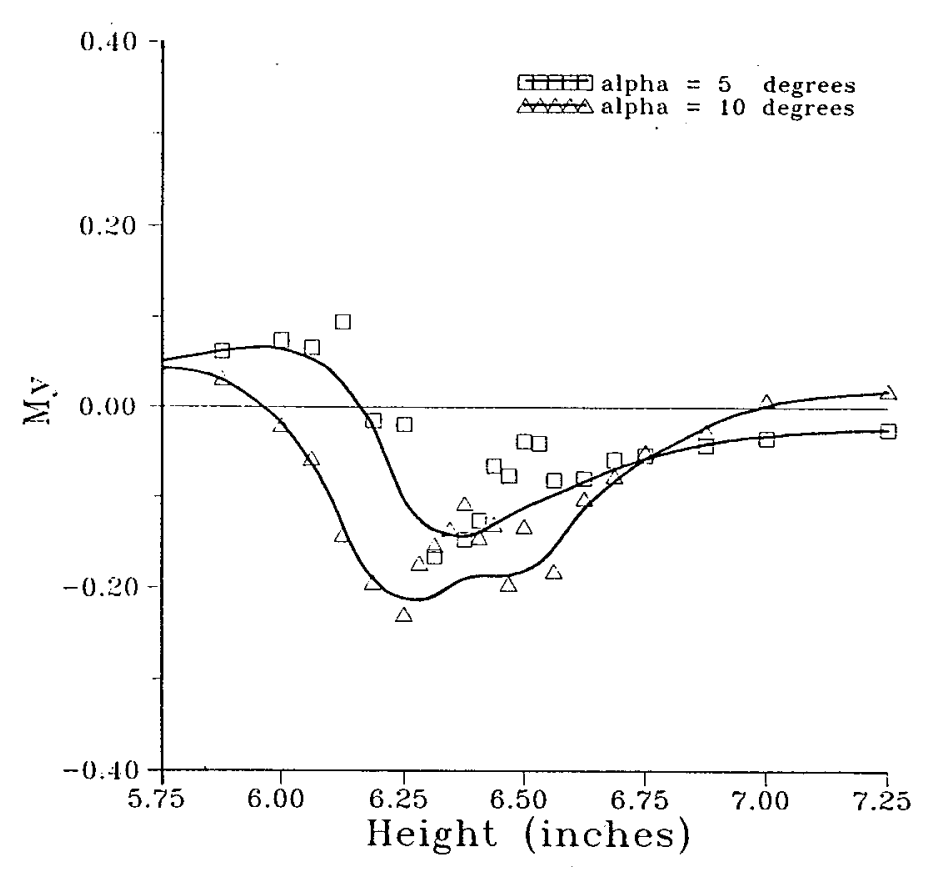

Figure 12(b) $-\mathrm{M}_{\mathrm{y}}$ Distribution

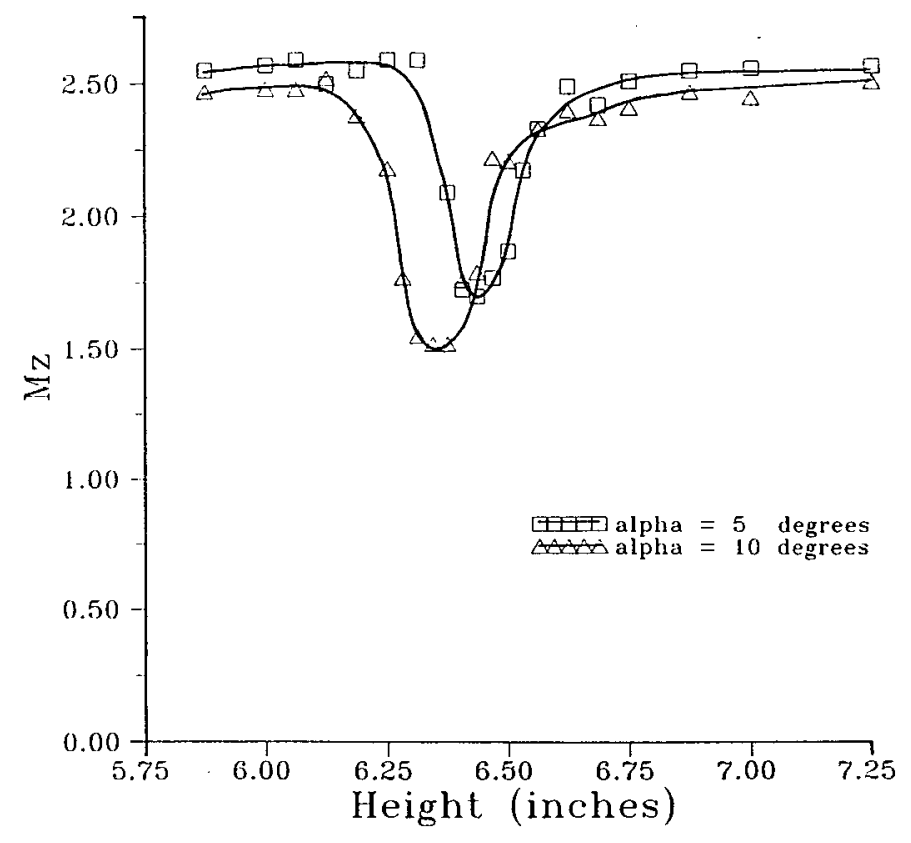

Figure $12(c)-M_{z}$ Distribution 

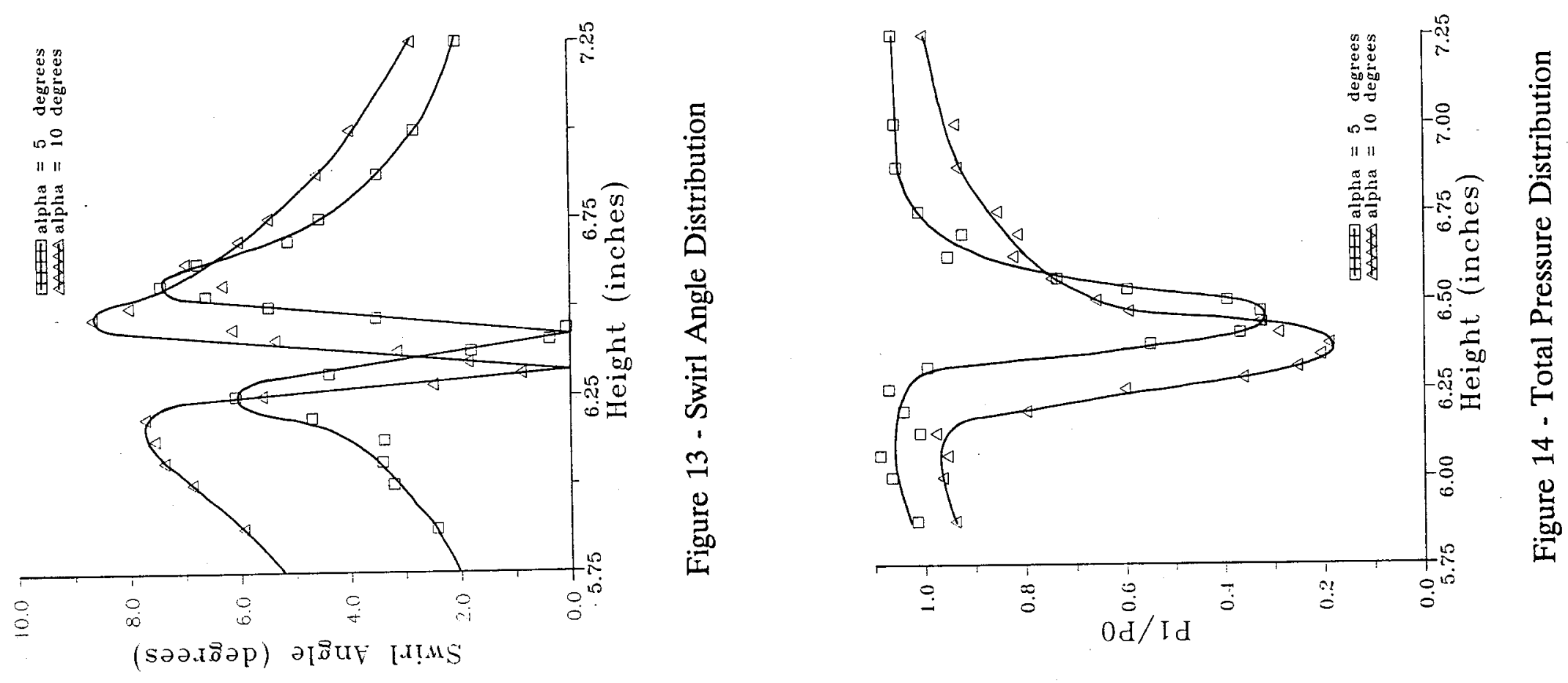